\title{
Quambalaria species associated with eucalypt diseases in southern China
}

\author{
Shuaifei CHEN ${ }^{1,2}$, Qianli LIU ${ }^{1}$, Guoqing LI ${ }^{1}$, Michael J. WINGFIELD (ه) ${ }^{2}$ \\ 1 China Eucalypt Research Centre, Chinese Academy of Forestry, Zhanjiang 524022, China \\ 2 Forestry and Agricultural Biotechnology Institute, University of Pretoria, Pretoria 0028, South Africa
}

\begin{abstract}
The genus Quambalaria includes several important pathogens of species of Eucalyptus and Corymbia, mainly causing leaf and shoot blight. Recently, extensive shoot and leaf dieback and stem cankers suspected to be Quambalaria diseases have been found on young Eucalyptus urophylla $\times$ E. grandis trees in Guangdong and Hainan Provinces. The occurrence of Quambalaria species and their association with eucalypt hosts within China needs to be investigated for tree diseases management. The isolates from the diseased samples were identified based on their morphological structures and phylogenetic analyses with DNA sequence data for the internal transcribed spacer region and large ribosome subunit RNA of the nuclear rDNA. This work revealed that three species of Quambalaria were present: Quambalaria pitereka from Corymbia citriodora, $Q$. eucalypti from $E$. urophylla $\times E$. grandis, both isolated from young eucalypt leaves and shoots in Guangdong Province, and Quambalaria simpsonii, which was isolated from stem cankers of $E$. urophylla $\times E$. grandis at four different sites across Guangdong and Hainan Provinces. These results confirmed that Quambalaria agents were associated with the diseases occurring on eucalypt hosts in South China. This is the first report of Q. eucalypti in Asia and the first report of $Q$. simpsonii in China on Eucalyptus trees.
\end{abstract}

Keywords Corymbia, Eucalyptus, forest pathogens, plantations, Myrtaceae

\section{Introduction}

In China, Eucalyptus spp. (Myrtaceae) have been widely established in commercial plantations which cover about 4.5 million hectares in southern China ${ }^{[1]}$. They include

Received May 2, 2017; accepted September 28, 2017

Correspondence: mike.wingfield@fabi.up.ac.za mainly cloned hybrids of Eucalyptus urophylla and E. grandis, other Eucalyptus species include E. camaldulensis, E. dunnii, E. globulus, E. pellita, E. smithii, E. urophylla, as well as their hybrids and clones ${ }^{[2-4]}$. Corymbia citriodora (Myrtaceae), previously classified as a species of Eucalyptus, has also been widely planted in southern China ${ }^{[2,3]}$ and the two genera are collectively referred to in this paper as eucalypts.

The extensive development of eucalypt plantations in China and the relatively limited numbers of clones planted in the past two decades has resulted in the appearance of numerous pests and pathogens that have caused increasing levels of damage ${ }^{[5]}$. Consequently, extensive surveys of eucalypt plantations have been undertaken in southern China, resulting in several important diseases being recorded. These include stem diseases caused by Teratosphaeria zuluensis ${ }^{[6,7]}$, species of Botryosphaeriaceae ${ }^{[8]}$, species of Cryphonectriaceae ${ }^{[9,10]}$ and Ceratocystis ${ }^{[11]}$. Leaf diseases caused by Calonectria spp. ${ }^{[12-14]}$, species of Mycosphaerellaceae and Teratosphaeriaceae $e^{[15,16]}$ have also emerged as serious problems. The leaf and shoot pathogen, Quambalaria pitereka has been found on Corymbia citriodora in the Guangdong Province of southern China ${ }^{[17]}$.

Six species of Quambalaria occur on eucalypts. They include $Q$. coyrecup, $Q$. cyanescens, $Q$. eucalypti, $Q$. pitereka, $Q$. pusilla and $Q$. simpsonii and all appear to be native to Australia where their host trees also occur naturally ${ }^{[18-27]}$. Quambalaria eucalypti has also been found on native Myrceugenia glaucescens (Myrtaceae) trees in Uruguay, although it seems likely to have been introduced into that country ${ }^{[28]}$. Of the six Quambalaria spp., $Q$. eucalypti and $Q$. pitereka cause leaf and shoot blight on eucalypts ${ }^{[20,21,24]}, Q$. coyrecup causes cankers and shoot blight on Corymbia spp. ${ }^{[23]}$, and $Q$. cyanescens is generally regarded as a saprophyte ${ }^{[22]}$. It remains unknown as to whether $Q$. simpsonii is pathogenic to eucalypts $^{[26]}$, and the taxonomic status of $Q$. pusilla remains unresolved ${ }^{[22,29]}$. 
Recently, leaf and shoot blight with symptoms typical of those caused by species of Quambalaria was observed on young $C$. citriodora and E. urophylla $\times E$. grandis trees in southern China. In addition, a fungus with morphological characteristics typical of Quambalaria was isolated from cankers on the stems of E. urophylla $\times$ E. grandis trees. The aims of this study were to identify these Quambalaria spp. based on comparisons of DNA sequence data and morphological characteristics.

\section{Materials and methods}

\subsection{Collections of fungal isolates}

Leaf and shoot blight was observed on $C$. citriodora trees of different provenances in two experimental plantations and an E. urophylla $\times E$. grandis plantation in Guangdong Province in southern China (Figs. 1a, 1c, 1d and 1e). White masses of conidia and conidiophores characteristic of the
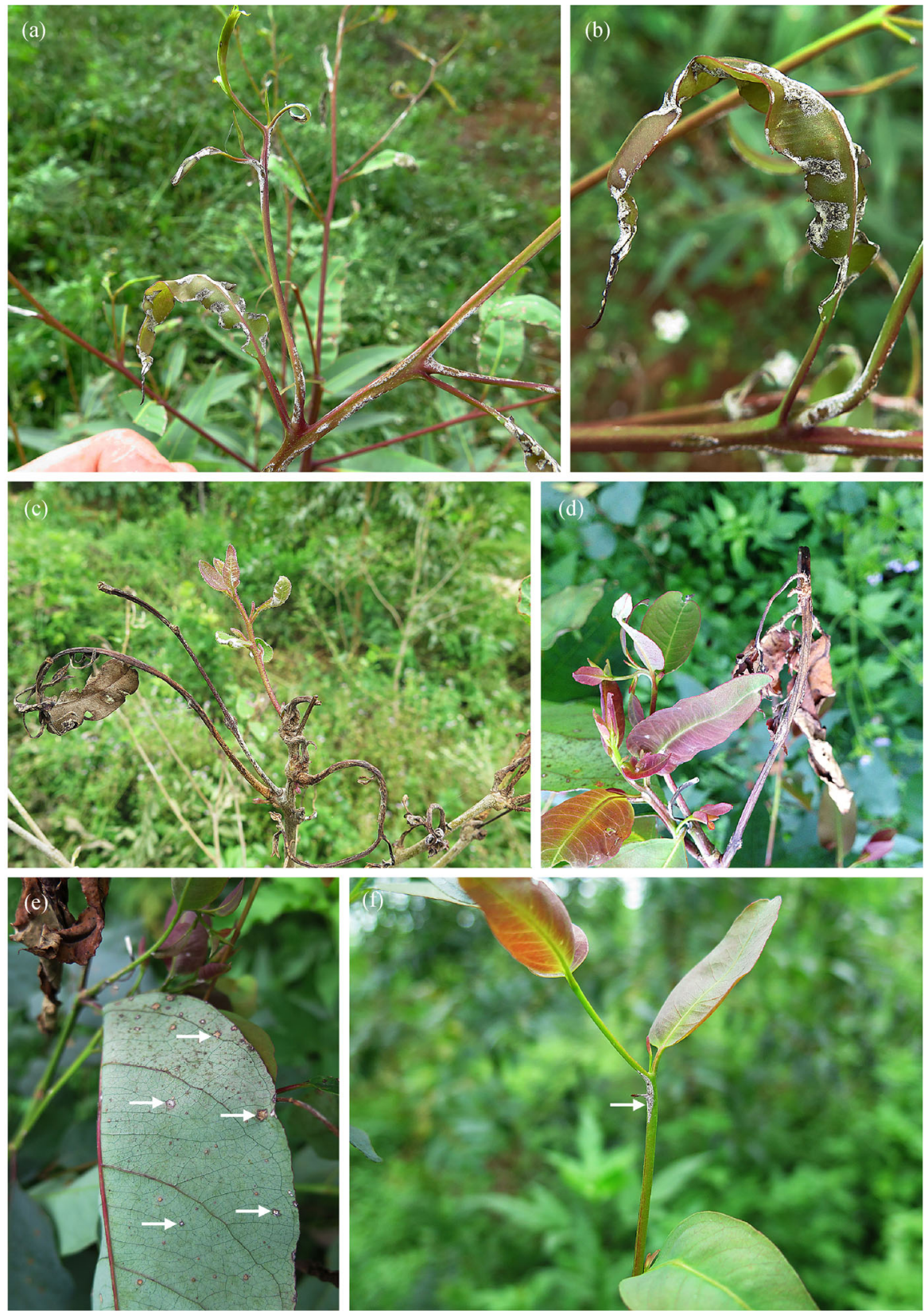

Fig. 1 Symptoms of infection by Quambalaria spp. on eucalypt trees. Shoot (a) and juvenile leave (b) of Corymbia citriodora infected by Quambalaria pitereka covered in white masses of conidia and conidiophores. New shoot (c) produced from the infected C. citriodora, and reinfected by $Q$. pitereka. Death of apical shoot (d) of Eucalyptus urophylla $\times$ E. grandis clone infected by Quambalaria simpsonii. Mature leaf (e) and young apical shoot (f) of E. urophylla $\times$ E. grandis clone infected by $Q$. simpsonii. Arrows indicate infected sites. 
Quambalaria $^{[21,22]}$ were common on the surface of the infected leaves and shoots (Figs. $1 \mathrm{~b}$ and 1f). Isolations were made by scraping conidial masses from the leaf and shoot surfaces and transferring these to $2 \%$ malt extract agar (MEA) medium (20 g malt extract and $20 \mathrm{~g}$ agar per liter water) and incubated at $25^{\circ} \mathrm{C}$. During the process of isolating the stem canker pathogen Teratosphaeria zuluensis (unpublished data) from cankered E. urophylla $\times$ $E$. grandis hybrid trees, a fungus with the morphological characteristics of Quambalaria species was isolated and these cultures were included in the present study. All Quambalaria isolates were collected during August 2015 and June 2016.

After the fungi had been cultured for $10 \mathrm{~d}$ on $2 \%$ MEA, single germ tubes emerging from colonies were subculture on $2 \%$ MEA media to obtain pure cultures. Cultures were deposited in the culture collection of the China Eucalypt Research Centre, Chinese Academy of Forestry, Zhanjiang, China. Representative isolates were also deposited at the China Forestry Culture Collection Centre, Beijing, China (Table 1).

\subsection{DNA extraction, PCR and sequence reactions}

Isolates collected from eucalypt trees in this study were identified based on DNA sequence comparisons (Table 1). For DNA extraction, isolates were grown on $2 \%$ MEA at $25^{\circ} \mathrm{C}$ for $10 \mathrm{~d}$ after which actively growing mycelium for each isolate was scraped from the surface of the medium using sterile scalpel blades and transferred to $1.5-\mathrm{mL}$ Eppendorf tubes. DNA was extracted using "method 5" described by Van Burik et al. ${ }^{[31]}$. The concentration of resulting DNA was checked using a Nano-Drop 2000 Spectrometer (Thermo Fisher Scientific Inc., Waltham, MA, USA).

Two gene regions, the internal transcribed spacer (ITS) regions including the $5.8 \mathrm{~S}$ gene of the rDNA operon and the conserved nuclear large subunit (LSU) rDNA were amplified as described by Chen et al. ${ }^{[32]}$. Nucleotide sequences were edited using MEGA version 4 software ${ }^{[33]}$. All sequences obtained in this study were deposited in GenBank (Table 1).

\subsection{Phylogenetic analyses}

To identify the isolates, sequences of ITS and LSU gene regions were compared to sequences of all described Quambalaria species, including the ex-type cultures of all the identified species from GenBank (Table 1). Also, in order to examine the diversity of the Quambalaria species, the haplotypes were determined from the polymorphic nucleotides within the aligned sequence data of ITS and LSU regions for isolates collected in this and previous studies.
To characterize the haplotypes from ITS sequences, all haplotypes designated by Pegg et al. ${ }^{[24]}$ were determined for all isolates of Quambalaria spp. from this and previous studies (Table 1). For phylogenetic analyses, two isolates representing each haplotype were used. Where only one isolate was available for a particular haplotype, this isolate was duplicated in the phylogenetic analyses to determine whether it would reside in an independent clade. Microstroma juglandis was used as the outgroup taxon (Table 1).

For haplotype determination using LSU sequences, representative Chinese isolates which included all the haplotypes determined based on the ITS sequences, and all isolates for which the LSU had been sequenced in previous studies were included (Table 1). All isolates used for haplotype determination by LSU sequences were used in the phylogenetic analyses. Where only one isolate was available for a particular haplotype, the isolates were duplicated in the phylogenetic analyses. M. juglandis was also used as the outgroup taxon for the LSU analyses (Table 1).

Sequences in ITS and LSU data sets were aligned using the iterative refinement method (FFT-NS-i settings) of the online platform of MAFFT v. $5.667^{[34]}$. The alignments were further edited manually in MEGA version 4 software $^{[33]}$. All alignments were deposited in TreeBASE.

The phylogenetic analyses were conducted using the maximum likelihood (ML) method, the ML tests were conducted with PHYML v. $3.0^{[35]}$ and the best models of nucleotide substitution were established with MODELTEST v. $3.7^{[36]}$. The analyses were conducted using PHYML v. $3.0^{[35]}$. Additional ML parameters in PHYML included retention of the maximum number of 1000 trees and the determination of nodal support by nonparametric bootstrapping with 1000 replicates. The phylogenetic trees were viewed using MEGA version 4 software $^{[33]}$.

\subsection{Morphology}

Single hyphal tip cultures of each Quambalaria sp. identified using DNA sequence data were subculture on $2 \%$ MEA media for 2 weeks at $25^{\circ} \mathrm{C}$ for morphological analysis. Four isolates for each identified Quambalaria sp. were used for comparisons of colony morphology. Conidiogenous cells and conidia were mounted in sterile water on microscope slides for measurements to be made using a Zeiss Axio Imager A1 microscope and a Zeiss AxioCam MRc digital camera with Zeiss Axio Vision Rel. 4.8 software (Carl Zeiss Ltd., Munchen, Germany). For each isolate, 25 measurements were made of conidia and ten of conidiophores. These measurements were compared with those published for species of Quambalaria. Results are presented as (minimum-) (mean - standard deviation) $-($ mean + standard deviation) $(-$ maximum $)$. 
Table 1 Isolates of Quambalaria species collected from eucalypt trees in southern China in 2015 and 2016 and used for phylogenetic and morphological analysis

\begin{tabular}{|c|c|c|c|c|c|c|c|}
\hline \multirow{2}{*}{ Identity } & \multirow{2}{*}{ Isolate No. ${ }^{a}$} & \multicolumn{2}{|c|}{ GenBank accession No. $^{\mathrm{b}}$} & \multirow{2}{*}{ Host } & \multirow{2}{*}{ Location } & \multirow{2}{*}{ Collector } & \multirow{2}{*}{ Reference } \\
\hline & & ITS & LSU & & & & \\
\hline $\begin{array}{l}\text { Quambalaria } \\
\text { coyrecup }\end{array}$ & WAC12947 & DQ823431 & DQ823444 & Corymbia calophylla & $\begin{array}{c}\text { Western Australia, } \\
\text { Australia }\end{array}$ & T Paap & Paap et al. ${ }^{[23]}$ \\
\hline Q. coyrecup & WAC $12948^{\text {de }}$ & DQ823433 & DQ823446 & C. calophylla & $\begin{array}{c}\text { Western Australia, } \\
\text { Australia }\end{array}$ & T Paap & Paap et al. ${ }^{[23]}$ \\
\hline Q. coyrecup & WAC $12949^{\mathrm{e}}$ & DQ823432 & DQ823445 & C. calophylla & $\begin{array}{c}\text { Western Australia, } \\
\text { Australia }\end{array}$ & T Paap & Paap et al. ${ }^{[23]}$ \\
\hline Q. coyrecup & WAC $12950^{\mathrm{de}}$ & DQ823429 & DQ823447 & C. calophylla & $\begin{array}{c}\text { Western Australia, } \\
\text { Australia }\end{array}$ & T Paap & Paap et al. ${ }^{[23]}$ \\
\hline Q. coyrecup & WAC12951 ${ }^{\mathrm{de}}$ & DQ823430 & DQ823448 & C. calophylla & $\begin{array}{c}\text { Western Australia, } \\
\text { Australia }\end{array}$ & T Paap & Paap et al. ${ }^{[23]}$ \\
\hline Q. coyrecup & BRIP48338 ${ }^{\mathrm{d}}$ & EF444877 & $\mathrm{N} / \mathrm{A}^{\mathrm{g}}$ & C. polycarpa & $\begin{array}{l}\text { Darwin, Northern Ter- } \\
\text { ritory, Australia }\end{array}$ & R Pitkethley & Pegg et al. ${ }^{[24]}$ \\
\hline Q. coyrecup & BRIP48339 & EF444878 & N/A & C. polycarpa & $\begin{array}{l}\text { Darwin, Northern Ter- } \\
\text { ritory, Australia }\end{array}$ & R Pitkethley & Pegg et al. ${ }^{[24]}$ \\
\hline Q. cyanescens & $\begin{array}{c}\mathrm{CBS} 357.73^{\text {cde }}= \\
\text { CMW5583 }\end{array}$ & DQ317622 & DQ317615 & skin of man & Netherlands & TF Visser & de Beer et al. ${ }^{[22]}$ \\
\hline Q. cyanescens & $\begin{array}{c}\mathrm{CBS} 876.73^{\mathrm{de}}= \\
\text { CMW5584 }\end{array}$ & DQ317623 & DQ317616 & Eucalyptus pauciflora & $\begin{array}{c}\text { New South Wales, } \\
\text { Australia }\end{array}$ & VF Brown & de Beer et al. ${ }^{[22]}$ \\
\hline Q. cyanescens & WAC12952 de & DQ823419 & DQ823440 & C. calophylla & $\begin{array}{c}\text { Western Australia, } \\
\text { Australia }\end{array}$ & T Paap & Paap et al. ${ }^{[23]}$ \\
\hline Q. cyanescens & WAC $12953^{\text {de }}$ & DQ823422 & DQ823443 & C. ficifolia & $\begin{array}{c}\text { Western Australia, } \\
\text { Australia }\end{array}$ & T Paap & Paap et al. ${ }^{[23]}$ \\
\hline Q. cyanescens & WAC $12954^{\mathrm{e}}$ & DQ823420 & DQ823442 & C. calophylla & $\begin{array}{c}\text { Western Australia, } \\
\text { Australia }\end{array}$ & T Paap & Paap et al. ${ }^{[23]}$ \\
\hline Q. cyanescens & WAC $12955^{\text {de }}$ & DQ823421 & DQ823441 & C. calophylla & $\begin{array}{c}\text { Western Australia, } \\
\text { Australia }\end{array}$ & T Paap & Paap et al. ${ }^{[23]}$ \\
\hline Q. cyanescens & BRIP48396 ${ }^{\mathrm{d}}$ & EF444874 & N/A & Native $C$. citriodora & $\begin{array}{l}\text { Beaudesert, Queens- } \\
\text { land, Australia }\end{array}$ & GS Pegg & Pegg et al. ${ }^{[24]}$ \\
\hline Q. cyanescens & BRIP48398 ${ }^{d}$ & EF444875 & N/A & Native $C$. citriodora & $\begin{array}{l}\text { Beaudesert, Queens- } \\
\text { land, Australia }\end{array}$ & GS Pegg & Pegg et al. ${ }^{[24]}$ \\
\hline Q. cyanescens & BRIP48403 ${ }^{\mathrm{d}}$ & EF444876 & N/A & Native $C$. citriodora & $\begin{array}{l}\text { Beaudesert, Queens- } \\
\text { land, Australia }\end{array}$ & GS Pegg & Pegg et al. ${ }^{[24]}$ \\
\hline Q. eucalypti & $\begin{array}{c}\mathrm{CBS} 118844^{\text {cde }}= \\
\text { CMW1101 }\end{array}$ & DQ317625 & DQ317618 & Eucalyptus grandis & $\begin{array}{c}\text { Kwambonambi, South } \\
\text { Africa }\end{array}$ & MJ Wingfield & de Beer et al. ${ }^{[22]}$ \\
\hline Q. eucalypti & $\begin{array}{c}\text { CBS } 119680^{\text {de }}= \\
\text { CMW11678 }\end{array}$ & DQ317626 & DQ317619 & $\begin{array}{l}\text { E. grandis clone } \\
\text { NH58 }\end{array}$ & $\begin{array}{c}\text { Kwambonambi, South } \\
\text { Africa }\end{array}$ & L Lombard & de Beer et al. ${ }^{[22]}$ \\
\hline Q. eucalypti & CMW14329 & DQ317614 & N/A & $\begin{array}{c}\text { E. grandis } \times E \text {. } \\
\text { camaldulensis clone }\end{array}$ & $\begin{array}{c}\text { Kwambonambi, South } \\
\text { Africa }\end{array}$ & J Roux & Roux et al. ${ }^{[30]}$ \\
\hline Q. eucalypti & $\begin{array}{c}\text { CBS118615= } \\
\text { CMW17252 }\end{array}$ & DQ317609 & N/A & E. nitens & $\begin{array}{c}\text { Rooihoogte, South } \\
\text { Africa }\end{array}$ & $\begin{array}{l}\text { ZL Mthalane \& J } \\
\text { Roux }\end{array}$ & Roux et al. ${ }^{[30]}$ \\
\hline Q. eucalypti & CMW17253 & DQ317610 & N/A & E. nitens & $\begin{array}{c}\text { Rooihoogte, South } \\
\text { Africa }\end{array}$ & $\begin{array}{l}\text { ZL Mthalane \& J } \\
\text { Roux }\end{array}$ & Roux et al. ${ }^{[30]}$ \\
\hline Q. eucalypti & CMW17254 & DQ317611 & N/A & E. nitens & $\begin{array}{c}\text { Rooihoogte, South } \\
\text { Africa }\end{array}$ & $\begin{array}{l}\text { ZL Mthalane \& J } \\
\text { Roux }\end{array}$ & Roux et al. ${ }^{[30]}$ \\
\hline Q. eucalypti & CMW17255 & DQ317612 & N/A & E. nitens & $\begin{array}{c}\text { Rooihoogte, South } \\
\text { Africa }\end{array}$ & $\begin{array}{l}\text { ZL Mthalane \& J } \\
\text { Roux }\end{array}$ & Roux et al. ${ }^{[30]}$ \\
\hline Q. eucalypti & $\begin{array}{c}\text { CBS118616= } \\
\text { CMW17771 }\end{array}$ & DQ317613 & N/A & E. grandis clone & $\begin{array}{c}\text { Kwambonambi, South } \\
\text { Africa }\end{array}$ & J Roux & Roux et al. ${ }^{[30]}$ \\
\hline Q. eucalypti & UY1036 & EU439922 & N/A & $\begin{array}{c}\text { Myrceugenia } \\
\text { glaucescens }\end{array}$ & Uruguay & C. A. Pérez & Pérez et al. ${ }^{[28]}$ \\
\hline
\end{tabular}


(Continued)

\begin{tabular}{|c|c|c|c|c|c|c|c|}
\hline \multirow{2}{*}{ Identity } & \multirow{2}{*}{ Isolate No. ${ }^{\mathrm{a}}$} & \multicolumn{2}{|c|}{ GenBank accession No. ${ }^{b}$} & \multirow{2}{*}{ Host } & \multirow{2}{*}{ Location } & \multirow{2}{*}{ Collector } & \multirow{2}{*}{ Reference } \\
\hline & & ITS & LSU & & & & \\
\hline Q. eucalypti & UY1718 & EU439923 & $\mathrm{N} / \mathrm{A}$ & M. glaucescens & Uruguay & C. A. Pérez & Pérez et al. ${ }^{[28]}$ \\
\hline Q. eucalypti & PE3/MEAN 996 & JX297605 & N/A & E. globulus & Portugal & N/A & Braganca et al. ${ }^{[27]}$ \\
\hline Q. eucalypti & PE6/MEAN 997 & JX297603 & N/A & E. globulus & Portugal & N/A & Braganca et al. ${ }^{[27]}$ \\
\hline Q. eucalypti & PE27/MEAN 998 & JX297604 & N/A & E. globulus & Portugal & N/A & Braganca et al. ${ }^{[27]}$ \\
\hline Q. eucalypti & PE28/MEAN 999 & JX297600 & N/A & E. globulus & Portugal & N/A & Braganca et al. ${ }^{[27]}$ \\
\hline Q. eucalypti & $\begin{array}{c}\text { PE29/MEAN } \\
1000\end{array}$ & JX297602 & N/A & E. globulus & Portugal & N/A & Braganca et al. ${ }^{[27]}$ \\
\hline Q. eucalypti & $\begin{array}{c}\text { PE30/MEAN } \\
1001\end{array}$ & JX297601 & N/A & E. globulus & Portugal & N/A & Braganca et al. ${ }^{[27]}$ \\
\hline Q. eucalypti & $\begin{array}{c}\text { PE52/MEAN } \\
1002\end{array}$ & JX297606 & N/A & E. globulus & Portugal & N/A & Braganca et al. ${ }^{[27]}$ \\
\hline Q. eucalypti & $\begin{array}{c}\text { PE53/MEAN } \\
1003\end{array}$ & JX297598 & N/A & E. globulus & Portugal & N/A & Braganca et al. ${ }^{[27]}$ \\
\hline Q. eucalypti & $\begin{array}{c}\text { PE54/MEAN } \\
1004\end{array}$ & JX297599 & N/A & E. globulus & Portugal & N/A & Braganca et al. ${ }^{[27]}$ \\
\hline Q. eucalypti & $\begin{array}{c}\text { PE93/MEAN } \\
1006\end{array}$ & KR336802 & N/A & E. globulus & Portugal & N/A & Braganca et al. ${ }^{[27]}$ \\
\hline Q. eucalypti & $\begin{array}{c}\text { PE96/MEAN } \\
1009\end{array}$ & KR336803 & N/A & E. globulus & Portugal & N/A & Braganca et al. ${ }^{[27]}$ \\
\hline Q. eucalypti & $\begin{array}{c}\text { PE151/MEAN } \\
1012\end{array}$ & KR336804 & N/A & E. globulus & Portugal & N/A & Braganca et al. ${ }^{[27]}$ \\
\hline Q. eucalypti & $\begin{array}{c}\text { PE152/MEAN } \\
1013\end{array}$ & KR336805 & N/A & E. globulus & Portugal & N/A & Braganca et al. ${ }^{[27]}$ \\
\hline Q. eucalypti & $\begin{array}{c}\text { PE153/MEAN } \\
1014\end{array}$ & KR336806 & N/A & E. globulus & Portugal & N/A & Braganca et al. ${ }^{[27]}$ \\
\hline Q. eucalypti & $\begin{array}{c}\text { PE154/MEAN } \\
1015\end{array}$ & KR336807 & N/A & E. globulus & Portugal & N/A & Braganca et al. ${ }^{[27]}$ \\
\hline Q. eucalypti & BRIP48367 & EF444823 & N/A & $\begin{array}{c}\text { C. torelliana } \times C \text {. } \\
\text { citriodora } \text { subsp. var- } \\
\text { iegata }\end{array}$ & $\begin{array}{l}\text { Walkamin, Queens- } \\
\text { land, Australia }\end{array}$ & GS Pegg & Pegg et al. ${ }^{[24]}$ \\
\hline Q. eucalypti & BRIP48422 ${ }^{\mathrm{d}}$ & EF444832 & N/A & E. dunnii & $\begin{array}{c}\text { New South Wales, } \\
\text { Australia }\end{array}$ & AJ Carnegie & Pegg et al. ${ }^{[24]}$ \\
\hline Q. eucalypti & BRIP48498 ${ }^{\mathrm{d}}$ & EF444844 & N/A & E. grandis & $\begin{array}{c}\text { New South Wales, } \\
\text { Australia }\end{array}$ & AJ Carnegie & Pegg et al. ${ }^{[24]}$ \\
\hline Q. eucalypti & BRIP48507 ${ }^{\mathrm{d}}$ & EF444822 & N/A & E. grandis & $\begin{array}{c}\text { Moggill, Queensland, } \\
\text { Australia }\end{array}$ & GS Pegg & Pegg et al. ${ }^{[24]}$ \\
\hline Q. eucalypti & $\operatorname{CERC8476}^{\mathrm{d}}$ & KY615009 & N/A & E. grandis & Guangdong, China & SF Chen \& JQ Li & This study \\
\hline Q. eucalypti & CERC8477 ${ }^{\mathrm{g}}$ & KY615010 & N/A & E. grandis & Guangdong, China & SF Chen \& JQ Li & This study \\
\hline Q. eucalypti & CERC8478 & KY615011 & N/A & E. grandis & Guangdong, China & SF Chen \& JQ Li & This study \\
\hline Q. eucalypti & CERC8479g $^{g}$ & KY615012 & KY615050 & E. grandis & Guangdong, China & SF Chen \& JQ Li & This study \\
\hline Q. eucalypti & CERC8480" & KY615013 & N/A & E. grandis & Guangdong, China & SF Chen \& JQ Li & This study \\
\hline Q. eucalypti & CERC8481 & KY615014 & KY615051 & E. grandis & Guangdong, China & SF Chen \& JQ Li & This study \\
\hline Q. eucalypti & CERC8482 $^{\mathrm{g}}$ & KY615015 & $\mathrm{N} / \mathrm{A}$ & E. grandis & Guangdong, China & SF Chen \& JQ Li & This study \\
\hline Q. eucalypti & CERC8483 & KY615016 & N/A & E. grandis & Guangdong, China & SF Chen \& JQ Li & This study \\
\hline Q. pitereka & DAR $19773^{\text {cde }}$ & DQ823423 & DQ823438 & C. eximia & $\begin{array}{c}\text { New South Wales, } \\
\text { Australia }\end{array}$ & $\begin{array}{c}\text { J Walker \& AL } \\
\text { Bertus }\end{array}$ & Paap et al. ${ }^{[23]}$ \\
\hline$Q$. pitereka & CMW $6707^{\mathrm{de}}$ & DQ317627 & DQ317620 & Corymbia maculata & $\begin{array}{c}\text { New South Wales, } \\
\text { Australia }\end{array}$ & MJ Wingfield & de Beer et al. ${ }^{[22]}$ \\
\hline
\end{tabular}


(Continued)

\begin{tabular}{|c|c|c|c|c|c|c|c|}
\hline \multirow{2}{*}{ Identity } & \multirow{2}{*}{ Isolate No. ${ }^{a}$} & \multicolumn{2}{|c|}{ GenBank accession No. ${ }^{b}$} & \multirow{2}{*}{ Host } & \multirow{2}{*}{ Location } & \multirow{2}{*}{ Collector } & \multirow{2}{*}{ Reference } \\
\hline & & ITS & LSU & & & & \\
\hline Q. pitereka & $\begin{array}{c}\mathrm{CBS}_{118828^{\mathrm{de}}}= \\
\text { CMW5318 }\end{array}$ & DQ317628 & DQ317621 & $\begin{array}{l}\text { C. citriodora subsp. } \\
\text { variegata }\end{array}$ & Queensland, Australia & M Ivory & de Beer et al. ${ }^{[22]}$ \\
\hline Q. pitereka & CMW23610 & EF427372 & N/A & C. citriodora & Guangdong, China & YJ Xie & Zhou et al. ${ }^{[17]}$ \\
\hline Q. pitereka & CMW23611 & EF427373 & N/A & C. citriodora & Guangdong, China & YJ Xie & Zhou et al. ${ }^{[17]}$ \\
\hline Q. pitereka & CMW23612 & EF427374 & N/A & C. citriodora & Guangdong, China & YJ Xie & Zhou et al. ${ }^{[17]}$ \\
\hline Q. pitereka & CMW23613 & EF427375 & N/A & C. citriodora & Guangdong, China & YJ Xie & Zhou et al. ${ }^{[17]}$ \\
\hline Q. pitereka & BRIP48325 & EF427366 & N/A & $\begin{array}{c}\text { C. citriodora subsp. } \\
\text { variegata }\end{array}$ & Queensland, Australia & GS Pegg & Zhou et al. ${ }^{[17]}$ \\
\hline Q. pitereka & BRIP48361 ${ }^{\mathrm{d}}$ & EF427367 & N/A & $\begin{array}{c}\text { C. citriodora subsp. } \\
\text { variegata }\end{array}$ & Queensland, Australia & GS Pegg & Zhou et al. ${ }^{[17]}$ \\
\hline Q. pitereka & BRIP48370 ${ }^{\mathrm{d}}$ & EF427368 & N/A & $\begin{array}{l}\text { C. citriodora subsp. } \\
\text { variegata }\end{array}$ & Queensland, Australia & GS Pegg & Zhou et al. ${ }^{[17]}$ \\
\hline Q. pitereka & BRIP48384 ${ }^{d}$ & EF427369 & N/A & $\begin{array}{c}\text { C. citriodora subsp. } \\
\text { variegata }\end{array}$ & Queensland, Australia & GS Pegg & Zhou et al. ${ }^{[17]}$ \\
\hline Q. pitereka & BRIP48386 ${ }^{\text {ed }}$ & EF427370 & N/A & $\begin{array}{l}\text { C. citriodora subsp. } \\
\text { variegata }\end{array}$ & Queensland, Australia & GS Pegg & Zhou et al. ${ }^{[17]}$ \\
\hline Q. pitereka & BRIP48531 ${ }^{\mathrm{d}}$ & EF427371 & N/A & $\begin{array}{c}\text { C. citriodora subsp. } \\
\text { variegata }\end{array}$ & Queensland, Australia & GS Pegg & Zhou et al. ${ }^{[17]}$ \\
\hline Q. pitereka & WAC $12957^{\mathrm{e}}$ & DQ823426 & DQ823437 & C. ficifolia & $\begin{array}{l}\text { Western Australia, } \\
\text { Australia }\end{array}$ & T Paap & Paap et al. ${ }^{[23]}$ \\
\hline Q. pitereka & WAC $12958^{\mathrm{e}}$ & DQ823427 & DQ823436 & C. calophylla & $\begin{array}{c}\text { Western Australia, } \\
\text { Australia }\end{array}$ & T Paap & Paap et al. ${ }^{[23]}$ \\
\hline Q. pitereka & $\mathrm{QP} 26^{\mathrm{e}}$ & DQ823424 & DQ823434 & $\begin{array}{c}\text { C. citriodora subsp. } \\
\text { variegata }\end{array}$ & Queensland, Australia & GS Pegg & Paap et al. ${ }^{[23]}$ \\
\hline Q. pitereka & $\mathrm{QP}^{\mathrm{de}}$ & DQ823425 & DQ823439 & $\begin{array}{l}\text { C. citriodora subsp. } \\
\text { variegata }\end{array}$ & Queensland, Australia & GS Pegg & Paap et al. ${ }^{[23]}$ \\
\hline Q. pitereka & BRIP48346 ${ }^{\mathrm{d}}$ & EF444845 & N/A & $\begin{array}{l}\text { C. citriodora subsp. } \\
\text { citriodora }\end{array}$ & $\begin{array}{l}\text { Davies Creek, Queens- } \\
\text { land, Australia }\end{array}$ & GS Pegg & Pegg et al. ${ }^{[24]}$ \\
\hline Q. pitereka & BRIP48317 & EF444854 & N/A & C. henryi & $\begin{array}{l}\text { Coolabunia, Queens- } \\
\text { land, Australia }\end{array}$ & GS Pegg & Pegg et al. ${ }^{[24]}$ \\
\hline Q. pitereka & BRIP48381 ${ }^{\mathrm{d}}$ & EF444858 & N/A & $\begin{array}{l}\text { C. citriodora subsp. } \\
\text { citriodora }\end{array}$ & $\begin{array}{c}\text { Silkwood, Queensland, } \\
\text { Australia }\end{array}$ & GS Pegg & Pegg et al. ${ }^{[24]}$ \\
\hline Q. pitereka & BRIP48383 ${ }^{\mathrm{d}}$ & EF444859 & N/A & $\begin{array}{l}\text { C. citriodora subsp. } \\
\text { variegata }\end{array}$ & $\begin{array}{l}\text { Beaudesert, Queens- } \\
\text { land, Australia }\end{array}$ & GS Pegg & Pegg et al. ${ }^{[24]}$ \\
\hline$Q$. pitereka & WAC $12956^{\text {d }}$ & DQ823428 & N/A & C. ficifolia & $\begin{array}{l}\text { Western Australia, } \\
\text { Australia }\end{array}$ & T Paap & $\begin{array}{l}\text { Paap et al. }{ }^{[23]}, \\
\text { Pegg et al. }{ }^{[24]}\end{array}$ \\
\hline Q. pitereka & BRIP48349 & EF444860 & N/A & $\begin{array}{c}\text { C. torelliana } \times C \text {. } \\
\text { citriodora subsp. var- } \\
\text { iegata }\end{array}$ & $\begin{array}{c}\text { Mareeba, Queensland, } \\
\text { Australia }\end{array}$ & GS Pegg & Pegg et al. ${ }^{[24]}$ \\
\hline Q. pitereka & BRIP48325 ${ }^{\mathrm{d}}$ & EF427366 & N/A & $\begin{array}{l}\text { C. citriodora subsp. } \\
\text { variegata }\end{array}$ & $\begin{array}{c}\text { Binjour, Queensland, } \\
\text { Australia }\end{array}$ & GS Pegg & Pegg et al. ${ }^{[24]}$ \\
\hline Q. pitereka & BRIP48328 & EF444872 & N/A & $\begin{array}{c}\text { Native } C \text {. citriodora } \\
\text { subsp. variegata }\end{array}$ & $\begin{array}{l}\text { Dilkoon, New South } \\
\text { Wales, Australia }\end{array}$ & GS Pegg & Pegg et al. ${ }^{[24]}$ \\
\hline Q. pitereka & BRIP48432 ${ }^{\mathrm{d}}$ & EF444873 & N/A & $\begin{array}{c}\text { C. citriodora subsp. } \\
\text { variegata }\end{array}$ & $\begin{array}{l}\text { Grafton, New South } \\
\text { Wales, Australia }\end{array}$ & GS Pegg & Pegg et al. ${ }^{[24]}$ \\
\hline Q. pitereka & CERC8486 $^{\mathrm{de}}$ & KY615017 & KY615052 & $\begin{array}{l}\text { C. citriodora prove- } \\
\text { nance CERC10 }\end{array}$ & Guangdong, China & SF Chen \& GQ Li & This study \\
\hline Q. pitereka & $\mathrm{CERC8488}^{\mathrm{e}}$ & KY615018 & KY615053 & $\begin{array}{l}\text { C. citriodora prove- } \\
\text { nance CERC12 }\end{array}$ & Guangdong, China & SF Chen \& GQ Li & This study \\
\hline
\end{tabular}


(Continued)

\begin{tabular}{|c|c|c|c|c|c|c|c|}
\hline \multirow{2}{*}{ Identity } & \multirow{2}{*}{ Isolate No. ${ }^{\mathrm{a}}$} & \multicolumn{2}{|c|}{ GenBank accession No. ${ }^{b}$} & \multirow{2}{*}{ Host } & \multirow{2}{*}{ Location } & \multirow{2}{*}{ Collector } & \multirow{2}{*}{ Reference } \\
\hline & & ITS & LSU & & & & \\
\hline Q. pitereka & CERC8489 & KY615019 & N/A & $\begin{array}{l}\text { C. citriodora prove- } \\
\text { nance CERC13 }\end{array}$ & Guangdong, China & SF Chen \& GQ Li & This study \\
\hline Q. pitereka & CERC8491 & KY615020 & N/A & $\begin{array}{l}\text { C. citriodora prove- } \\
\text { nance CERC15 }\end{array}$ & Guangdong, China & SF Chen \& GQ Li & This study \\
\hline Q. pitereka & CERC8494 ${ }^{\mathrm{eg}}$ & KY615021 & KY615054 & $\begin{array}{l}\text { C. citriodora prove- } \\
\text { nance CERC17 }\end{array}$ & Guangdong, China & SF Chen \& GQ Li & This study \\
\hline Q. pitereka & CERC9093 & KY615022 & N/A & $\begin{array}{l}\text { C. citriodora prove- } \\
\text { nance CR76 }\end{array}$ & Guangdong, China & SF Chen \& Y Lin & This study \\
\hline$Q$. pitereka & CERC9094 & KY615023 & N/A & $\begin{array}{l}\text { C. citriodora prove- } \\
\text { nance N371 }\end{array}$ & Guangdong, China & SF Chen \& Y Lin & This study \\
\hline Q. pitereka & CERC9095 & KY615024 & N/A & $\begin{array}{l}\text { C. citriodora prove- } \\
\text { nance } \mathrm{N} 28\end{array}$ & Guangdong, China & SF Chen \& Y Lin & This study \\
\hline Q. pitereka & CERC9096 & KY615025 & N/A & $\begin{array}{l}\text { C. citriodora prove- } \\
\text { nance N411 }\end{array}$ & Guangdong, China & SF Chen \& Y Lin & This study \\
\hline Q. pitereka & CERC9097 ${ }^{\text {eg }}$ & KY615026 & KY615055 & $\begin{array}{l}\text { C. citriodora prove- } \\
\text { nance } \mathrm{N} 223\end{array}$ & Guangdong, China & SF Chen \& Y Lin & This study \\
\hline Q. pitereka & CERC9098 $^{\mathrm{g}}$ & KY615027 & N/A & $\begin{array}{l}\text { C. citriodora prove- } \\
\text { nance } \mathrm{N} 322\end{array}$ & Guangdong, China & SF Chen \& Y Lin & This study \\
\hline Q. pitereka & CERC9099 $^{\text {eg }}$ & KY615028 & KY615056 & $\begin{array}{l}\text { C. citriodora prove- } \\
\text { nance CR } 033\end{array}$ & Guangdong, China & SF Chen \& Y Lin & This study \\
\hline Q. pitereka & CERC9100 & KY615029 & N/A & $\begin{array}{l}\text { C. citriodora prove- } \\
\text { nance CR039 }\end{array}$ & Guangdong, China & SF Chen \& Y Lin & This study \\
\hline Q. pitereka & CERC9101 & KY615030 & N/A & $\begin{array}{l}\text { C. citriodora prove- } \\
\text { nance CR92 }\end{array}$ & Guangdong, China & SF Chen \& Y Lin & This study \\
\hline Q. pitereka & CERC9102 & KY615031 & N/A & $\begin{array}{l}\text { C. citriodora prove- } \\
\text { nance CR36 }\end{array}$ & Guangdong, China & SF Chen \& Y Lin & This study \\
\hline Q. pitereka & CERC9103 ${ }^{\mathrm{e}}$ & KY615032 & KY615057 & $\begin{array}{l}\text { C. citriodora prove- } \\
\text { nance N601 }\end{array}$ & Guangdong, China & SF Chen \& Y Lin & This study \\
\hline Q. pitereka & CERC9104 & KY615033 & N/A & $\begin{array}{l}\text { C. citriodora prove- } \\
\text { nance } \mathrm{N} 28\end{array}$ & Guangdong, China & SF Chen \& Y Lin & This study \\
\hline Q. simpsonii & CBS $124772^{\text {de }}$ & GQ303290 & GQ303321 & Eucalyptus tintinnans & Edith Falls, Australia & BA Summerell & Cheewangkoon et al. ${ }^{[26]}$ \\
\hline Q. simpsonii & CBS $124773^{\text {de }}$ & GQ303291 & GQ303322 & Eucalyptus sp. & Lamphoon, Thailand & R Cheewangkoon & Cheewangkoon et al. ${ }^{[26]}$ \\
\hline Q. simpsonii & CERC8496 $^{\text {dg }}$ & KY615034 & N/A & $\begin{array}{l}\text { E. urophylla } \times \\
\text { E. grandis }\end{array}$ & Hainan, China & SF Chen \& QL Liu & This study \\
\hline Q. simpsonii & CERC8499 & KY615035 & N/A & $\begin{array}{l}\text { E. urophylla } \times \\
\text { E. grandis }\end{array}$ & Hainan, China & SF Chen \& QL Liu & This study \\
\hline Q. simpsonii & $\operatorname{CERC8505}^{\mathrm{d}}$ & KY615036 & N/A & $\begin{array}{l}\text { E. urophylla } \times \\
\text { E. grandis }\end{array}$ & Hainan, China & SF Chen \& QL Liu & This study \\
\hline Q. simpsonii & CERC8507 $7^{\text {de }}$ & KY615037 & KY615058 & $\begin{array}{l}\text { E. urophylla } \times \\
\text { E. grandis }\end{array}$ & Hainan, China & SF Chen \& QL Liu & This study \\
\hline Q. simpsonii & CERC8512 $^{\mathrm{d}}$ & KY615038 & N/A & $\begin{array}{l}\text { E. urophylla } \times \\
\text { E. grandis }\end{array}$ & Hainan, China & SF Chen \& QL Liu & This study \\
\hline Q. simpsonii & CERC8514 & KY615039 & N/A & $\begin{array}{l}\text { E. urophylla } \times \\
\text { E. grandis }\end{array}$ & Hainan, China & SF Chen \& QL Liu & This study \\
\hline Q. simpsonii & CERC8516 & KY615040 & N/A & $\begin{array}{l}\text { E. urophylla } \times \\
\text { E. grandis }\end{array}$ & Hainan, China & SF Chen \& QL Liu & This study \\
\hline Q. simpsonii & CERC8517 $7^{\mathrm{e}}$ & KY615041 & KY615059 & $\begin{array}{l}\text { E. urophylla } \times \\
\text { E. grandis }\end{array}$ & Hainan, China & SF Chen \& QL Liu & This study \\
\hline Q. simpsonii & CERC8519 $^{\mathrm{dg}}$ & KY615042 & N/A & $\begin{array}{l}\text { E. urophylla } \times \\
\text { E. grandis }\end{array}$ & Hainan, China & SF Chen \& QL Liu & This study \\
\hline
\end{tabular}


(Continued)

\begin{tabular}{|c|c|c|c|c|c|c|c|}
\hline \multirow{2}{*}{ Identity } & \multirow{2}{*}{ Isolate No. $^{\mathrm{a}}$} & \multicolumn{2}{|c|}{ GenBank accession No. ${ }^{\text {b }}$} & \multirow{2}{*}{ Host } & \multirow{2}{*}{ Location } & \multirow{2}{*}{ Collector } & \multirow{2}{*}{ Reference } \\
\hline & & ITS & LSU & & & & \\
\hline Q. simpsonii & CERC8526 & KY615043 & $\mathrm{N} / \mathrm{A}$ & $\begin{array}{c}\text { E. urophylla } \times \\
\text { E. grandis }\end{array}$ & Hainan, China & SF Chen \& QL Liu & This study \\
\hline Q. simpsonii & CERC8532 & KY615044 & N/A & $\begin{array}{c}\text { E. urophylla } \times \\
\text { E. grandis }\end{array}$ & Guangdong, China & SF Chen \& JQ Li & This study \\
\hline Q. simpsonii & CERC8534 $^{\text {deg }}$ & KY615045 & KY615060 & $\begin{array}{c}\text { E. urophylla } \times \\
\text { E. grandis }\end{array}$ & Guangdong, China & SF Chen \& JQ Li & This study \\
\hline Q. simpsonii & CERC8536 $^{\mathrm{e}}$ & KY615046 & KY615061 & $\begin{array}{c}\text { E. urophylla } \times \\
\text { E. grandis }\end{array}$ & Guangdong, China & SF Chen \& JQ Li & This study \\
\hline Q. simpsonii & CERC8539 $^{\text {eg }}$ & KY615047 & KY615062 & $\begin{array}{c}\text { E. urophylla } \times \\
\text { E. grandis }\end{array}$ & Guangdong, China & SF Chen \& JQ Li & This study \\
\hline Q. simpsonii & CERC8541 $^{\mathrm{d}}$ & KY615048 & N/A & $\begin{array}{l}\text { E. urophylla } \times \\
\text { E. grandis }\end{array}$ & Guangdong, China & SF Chen \& JQ Li & This study \\
\hline Q. simpsonii & CERC8543 $^{\mathrm{d}}$ & KY615049 & N/A & $\begin{array}{c}\text { E. urophylla } \times \\
\text { E. grandis }\end{array}$ & Guangdong, China & SF Chen \& JQ Li & This study \\
\hline $\begin{array}{l}\text { Microstroma } \\
\text { juglandis }\end{array}$ & R.B. $2042^{\mathrm{de}}$ & DQ317634 & DQ317617 & Juglans regia & Germany & R Bauer & de Beer et al. ${ }^{[22]}$ \\
\hline
\end{tabular}

Note: ${ }^{a}$ Designation of isolates and culture collections: WAC, Department of Agriculture Western Australia Plant Pathogen Collection, Perth, Australia; BRIP, the plant pathology herbarium for Queensland Department of Primary Industries and Fisheries, Australia; CBS, Centraalbureau voor Schimmelcultures, Utrecht, The Netherlands; CMW, Tree Protection Co-operative Program, Forestry and Agricultural Biotechnology Institute, University of Pretoria, South Africa; CERC, China Eucalypt Research Centre (CERC), Chinese Academy of Forestry (CAF), ZhanJiang, GuangDong, China; DAR, the plant pathology herbarium for the Department of Agriculture in NSW, Australia; MEAN, fungal collection of Instituto Nacional de Investigação Agrária e Veterinária - INIAV, Oeiras, Portugal; R.B., Herbarium R. Bauer, Tübingen, Germany; Isolate numbers in boldface were collected in this study; ${ }^{\mathrm{b}}$ GenBank numbers in boldface were sequenced in this study; ${ }^{\mathrm{c}}$ Holotype specimens or ex-type isolates; ${ }^{\mathrm{d}}$ Isolates used in phylogenetic analyses by ITS sequence; ${ }^{\mathrm{e}}$ Isolates used in phylogenetic analyses by LSU sequence; ${ }^{\mathrm{f}} \mathrm{N} / \mathrm{A}=\mathrm{not}$ available; ${ }^{\mathrm{g}}$ Isolates used in morphological studies.

\section{Results}

\subsection{Collections of fungal isolates}

A total of 41 fungal isolates showing typical morphology of Quambalaria species were isolated. Seventeen isolates were from leaves or shoots on 17 C. citriodora trees of 16 provenances in two experimental plantations in Guangdong Province, eight isolates were from leaves of one E. urophylla $\times E$. grandis clone in one plantation in Guangdong Province, and 16 isolates were from cankers caused by $T$. zuluensis on the stems of E. urophylla $\times$ E. grandis clones in four plantations in Guangdong and Guangxi. Each of the 41 isolates was from a single tree and all were included in the DNA sequence comparisons and phylogenetic analyses (Table 1).

\subsection{Phylogenetic analyses}

The aligned ITS sequence data set consisted of 65 taxa and 634 characters (TreeBASE No. 20574). For the ML analyses, the Model Test analysis recommended a HKY $+\mathrm{I}+\mathrm{G}$ model [Lset Base $=(0.2639,0.2186,0.2071)$; Nst (number of substitution rate categories) $=2$; Transition/ transversion ratio $=2.6045$; Rate matrix $=(1.0000,4.3151$, $2.9747,2.9747,8.1747) ;$ Rates $=$ gamma; Shape $=$ 0.7544]. The phylogenetic analyses showed that isolates sequenced in this study resided in three clades that represent $Q$. pitereka, $Q$. eucalypti and $Q$. simpsonii (Fig. 2).

For the ITS sequences, all Chinese and all those from previous studies represented 32 haplotypes. These included three, seven, four, 12 and six haplotypes of $Q$. coyrecup, $Q$. cyanescens, $Q$. eucalypti, $Q$. pitereka Q. simpsonii, respectively (Tables $2-4, \mathrm{~S} 1$ ). The Chinese isolates collected in this study represented six haplotypes including one of Q. pitereka, one of Q. eucalypti, and four newly designated haplotypes of $Q$. simpsonii (Table $\mathrm{S} 1$ ).

The aligned LSU sequence data set consisted of 37 taxa and 561 characters (TreeBASE No. 20574). For ML analyses, model test analysis recommended a $\operatorname{TrN}+\mathrm{G}$ model [Lset Base $=(0.2492,0.1916,0.3025) ; \mathrm{Nst}=6$; Rate matrix $=(1.0000,7.7487,1.0000,1.0000,31.1002) ;$ Rates $=$ equal]. The phylogenetic analyses showed that isolates sequenced in this study resided in three clades of $Q$. pitereka, $Q$. eucalypti and $Q$. simpsonii, respectively (Fig. 3).

For the LSU sequences, 13 Chinese isolates which included all six haplotypes determined based on ITS sequences were used for phylogenetic analyses. These isolates and all of those sequenced in previous studies represented six haplotypes. These included two haplotypes of $Q$. pitereka and one each of $Q$. coyrecup, $Q$. cyanescens, $Q$. eucalypti and $Q$. simpsonii (Table $\mathrm{S} 1$ ). The Chinese isolates included in this study represented three haplotypes including one newly designated haplotype of 


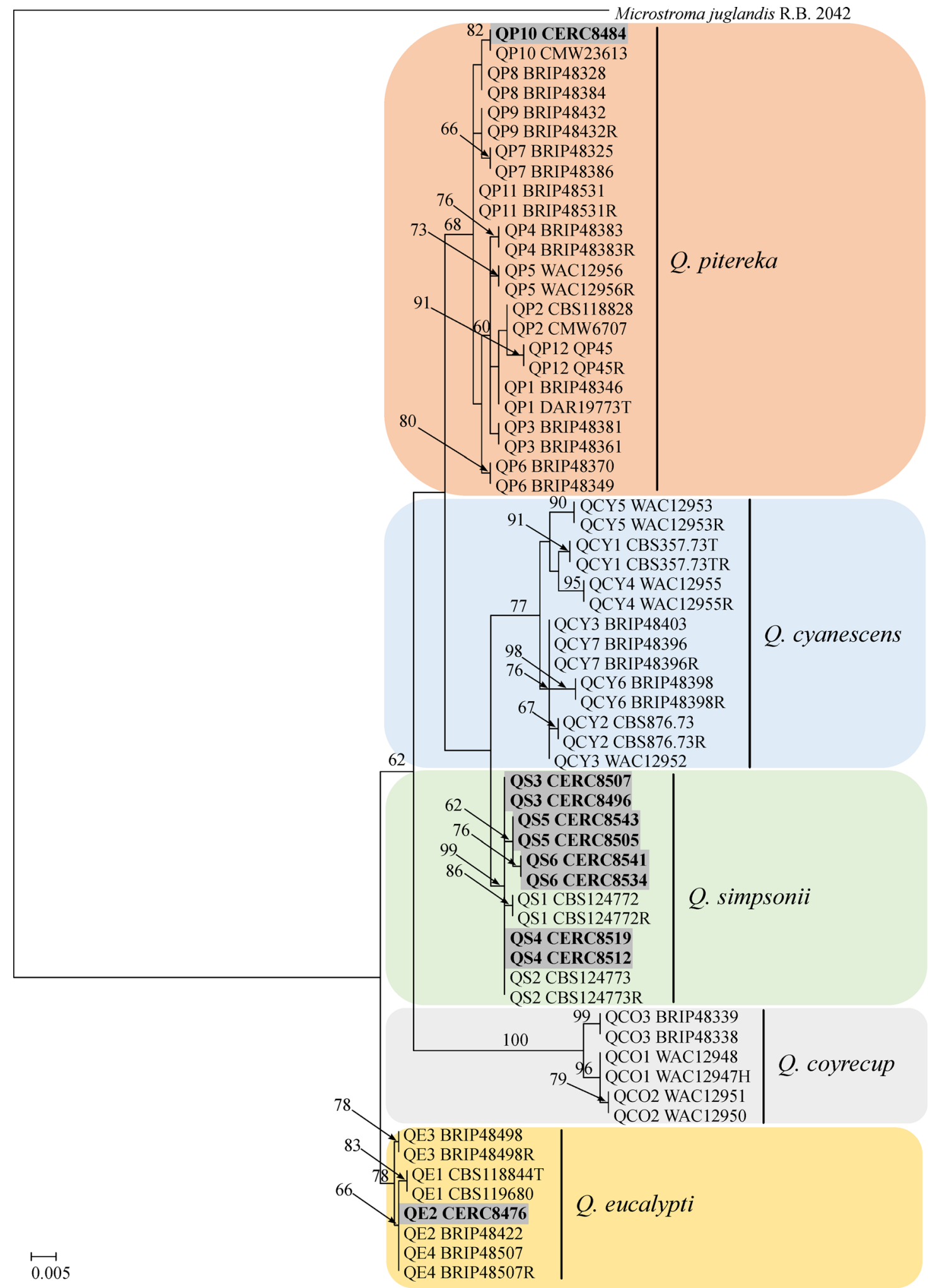

Fig. 2 Phylogenetic tree based on maximum likelihood analysis of ITS sequence data of haplotypes of five Quambalaria species, Q. coyrecup (QCO), Q. cyanescens (QCY), Q. eucalypti (QE), Q. pitereka (QP) and Q. simpsonii (QS). Bootstrap values $>60 \%$ are presented at branches, bootstrap values $<60 \%$ or absent values are not shown. Haplotypes and isolates from eucalypts in this study are in boldface and highlighted. Isolates representing ex-type are marked with $\mathrm{T}$, isolates repeated are marked with $\mathrm{R}$. The tree is rooted to Microstroma juglandis. 
Table 2 Four haplotypes of $Q$. eucalypti as determined from the polymorphic nucleotides within the aligned sequence data of ITS region for isolates collected from species of Eucalyptus, C. torelliana $\times$ C. citriodora subsp. variegate and M. glaucescens

\begin{tabular}{lccccccc}
\hline Haplotype & $121^{\mathrm{a}}$ & 158 & 159 & 160 & 161 & 162 & 558 \\
\hline QE1 & $\mathrm{T}$ & - & - & - & - & - & - \\
QE2 & $\mathrm{T}$ & - & - & - & - & - & $\mathrm{C}$ \\
QE3 & $\underline{\mathbf{C}}$ & - & - & - & - & - & $\mathrm{C}$ \\
QE4 & $\mathrm{T}$ & $\underline{\mathbf{T}}$ & $\underline{\mathbf{T}}$ & $\underline{\mathbf{A}}$ & $\underline{\mathbf{T}}$ & $\underline{\mathbf{A}}$ & $\mathbf{C}$ \\
\hline
\end{tabular}

Note: ${ }^{\text {a }}$ Base pair (bp) positions in aligned data; ${ }^{\mathrm{b}}$ Nucleotides that are different from the majority consensus sequence are underlined and highlighted in bold.

Table 3 Twelve haplotypes of $Q$. pitereka as determined from the polymorphic nucleotides within the aligned sequence data of ITS region for isolates collected from species of Corymbia

\begin{tabular}{|c|c|c|c|c|c|c|c|c|c|c|c|c|}
\hline Haplotype & $24^{a}$ & 54 & 107 & 112 & 214 & 219 & 233 & 236 & 390 & 451 & 606 & 614 \\
\hline$\overline{\mathrm{QP} 1}$ & $\mathrm{~T}$ & $\mathrm{~A}$ & $\mathrm{G}$ & $\mathrm{G}$ & $\mathrm{T}$ & $\underline{G}^{b}$ & $\mathrm{~T}$ & $\mathrm{C}$ & $\mathrm{C}$ & $\mathrm{C}$ & $\mathrm{C}$ & A \\
\hline QP2 & $\mathrm{T}$ & A & G & $\underline{\mathbf{A}}$ & $\mathrm{T}$ & $\underline{\mathbf{G}}$ & $\mathrm{T}$ & $\mathrm{C}$ & $\mathrm{C}$ & $\mathrm{C}$ & $\mathrm{C}$ & A \\
\hline QP3 & $\mathrm{T}$ & A & G & $\underline{\mathbf{A}}$ & $\mathrm{T}$ & A & $\mathrm{T}$ & $\mathrm{C}$ & $\mathrm{C}$ & $\mathrm{C}$ & $\mathrm{C}$ & A \\
\hline QP4 & $\mathrm{T}$ & A & G & G & $\mathrm{T}$ & A & $\mathrm{T}$ & $\mathrm{C}$ & $\underline{T}$ & $\mathrm{C}$ & $\mathrm{C}$ & A \\
\hline QP5 & $\mathrm{T}$ & A & G & G & $\mathrm{T}$ & A & $\mathrm{T}$ & $\mathrm{C}$ & $\mathrm{C}$ & $\underline{\mathbf{A}}$ & $\mathrm{C}$ & A \\
\hline QP6 & $\mathrm{T}$ & A & G & G & $\mathrm{T}$ & A & $\mathrm{T}$ & $\mathrm{C}$ & $\mathrm{C}$ & $\mathrm{C}$ & $\underline{\mathbf{T}}$ & G \\
\hline QP7 & $\underline{\mathbf{A}}$ & G & G & G & $\underline{\mathbf{G}}$ & A & $\mathrm{T}$ & $\mathrm{C}$ & $\mathrm{C}$ & $\mathrm{C}$ & $\mathrm{C}$ & G \\
\hline QP8 & $\underline{\mathbf{A}}$ & G & G & G & $\mathrm{T}$ & A & $\mathrm{T}$ & $\mathrm{C}$ & $\mathrm{C}$ & $\mathrm{C}$ & $\mathrm{C}$ & G \\
\hline QP9 & $\mathrm{T}$ & G & G & G & $\underline{\mathbf{G}}$ & A & $\mathrm{T}$ & $\mathrm{C}$ & $\mathrm{C}$ & $\mathrm{C}$ & $\mathrm{C}$ & G \\
\hline QP10 & $\underline{\mathbf{A}}$ & G & $\underline{\mathbf{A}}$ & G & $\mathrm{T}$ & A & $\mathrm{T}$ & $\mathrm{C}$ & $\mathrm{C}$ & $\mathrm{C}$ & $\mathrm{C}$ & G \\
\hline QP11 & $\mathrm{T}$ & G & G & G & $\mathrm{T}$ & A & $\mathrm{T}$ & $\mathrm{C}$ & $\mathrm{C}$ & $\mathrm{C}$ & $\mathrm{C}$ & G \\
\hline QP12 & $\mathrm{T}$ & G & G & $\underline{\mathbf{A}}$ & $\mathrm{T}$ & $\underline{\mathbf{G}}$ & $\underline{\mathrm{C}}$ & $\underline{\mathbf{G}}$ & $\mathrm{C}$ & $\mathrm{C}$ & $\mathrm{C}$ & A \\
\hline
\end{tabular}

Note: ${ }^{a}$ Base pair (bp) positions in aligned data; ${ }^{\mathrm{b}}$ Nucleotides that are different from the majority consensus sequence are underlined and highlighted in bold.

Table 4 Six haplotypes of $Q$. simpsonii as determined from the polymorphic nucleotides within the aligned sequence data of ITS region for isolates collected from species of Eucalyptus

\begin{tabular}{|c|c|c|c|c|c|}
\hline Haplotype & $4^{a}$ & 171 & 553 & 605 & 621 \\
\hline$\overline{\mathrm{QS} 1}$ & $\underline{\mathbf{A}}^{\mathrm{b}}$ & $\mathrm{A}$ & $\underline{T}$ & $\mathrm{~T}$ & - \\
\hline QS2 & $\underline{\mathbf{A}}$ & A & $\mathrm{C}$ & $\mathrm{T}$ & - \\
\hline QS3 & G & A & $\mathrm{C}$ & $\mathrm{T}$ & $\underline{\mathbf{T}}$ \\
\hline QS4 & G & A & $\mathrm{C}$ & $\mathrm{T}$ & - \\
\hline QS5 & G & A & $\mathrm{C}$ & $\underline{\mathbf{C}}$ & - \\
\hline QS6 & G & $\mathbf{G}$ & C & C & - \\
\hline
\end{tabular}

Note: ${ }^{a}$ Base pair (bp) positions in aligned data; ${ }^{\mathrm{b}}$ Nucleotides that are different from the majority consensus sequence are underlined and highlighted in bold.

Q. pitereka, and one haplotype for each of Q. eucalypti and Q. simpsonii (Table $\mathrm{S} 1$ ).

\subsection{Morphology}

Four isolates of Q. pitereka (CERC8494, CERC9097, CERC9098 and CERC9099), Q. eucalypti (CERC8477, CERC8479, CERC8480 and CERC8482) and Q. simpsonii (CERC8496, CERC8519, CERC8534 and CERC8539) were used in the morphological analysis. Colonies of these species were finely floccose becoming powdery and white
(Figs. 4a, 4c and 4e). The morphological characteristics of the fruiting structures of these species are summarized in Table 5 and illustrated in Figs. 4b, 4d and 4f. Conidiogenous cells of $Q$. pitereka, $Q$. eucalypti and Q. simpsonii were (7.4-89.6) $\mu \mathrm{m} \times(1.4-2.6) \mu \mathrm{m}$ (av. $46.0 \mu \mathrm{m} \times 2.0 \mu \mathrm{m}),(8.4-77.1) \mu \mathrm{m} \times(1.3-2.8) \mu \mathrm{m}$ (av. $37.4 \mu \mathrm{m} \times 2.2 \mu \mathrm{m}$ ), and ( 7.0-82.1) $\mu \mathrm{m} \times(1.5-2.9)$ $\mu \mathrm{m}$ (av. $25.6 \mu \mathrm{m} \times 2.4 \mu \mathrm{m})$, respectively. The conidia of $Q$. pitereka (primary conidia narrow fusiform, av. $10.9 \mu \mathrm{m}$ $\times 3.4 \mu \mathrm{m}$, length $/$ width $=3.2$; secondary conidia narrow fusiform, av. $6.0 \mu \mathrm{m} \times 2.7 \mu \mathrm{m}$, length/width $=2.2$ ) are longer and narrower (by length/width) than that of 


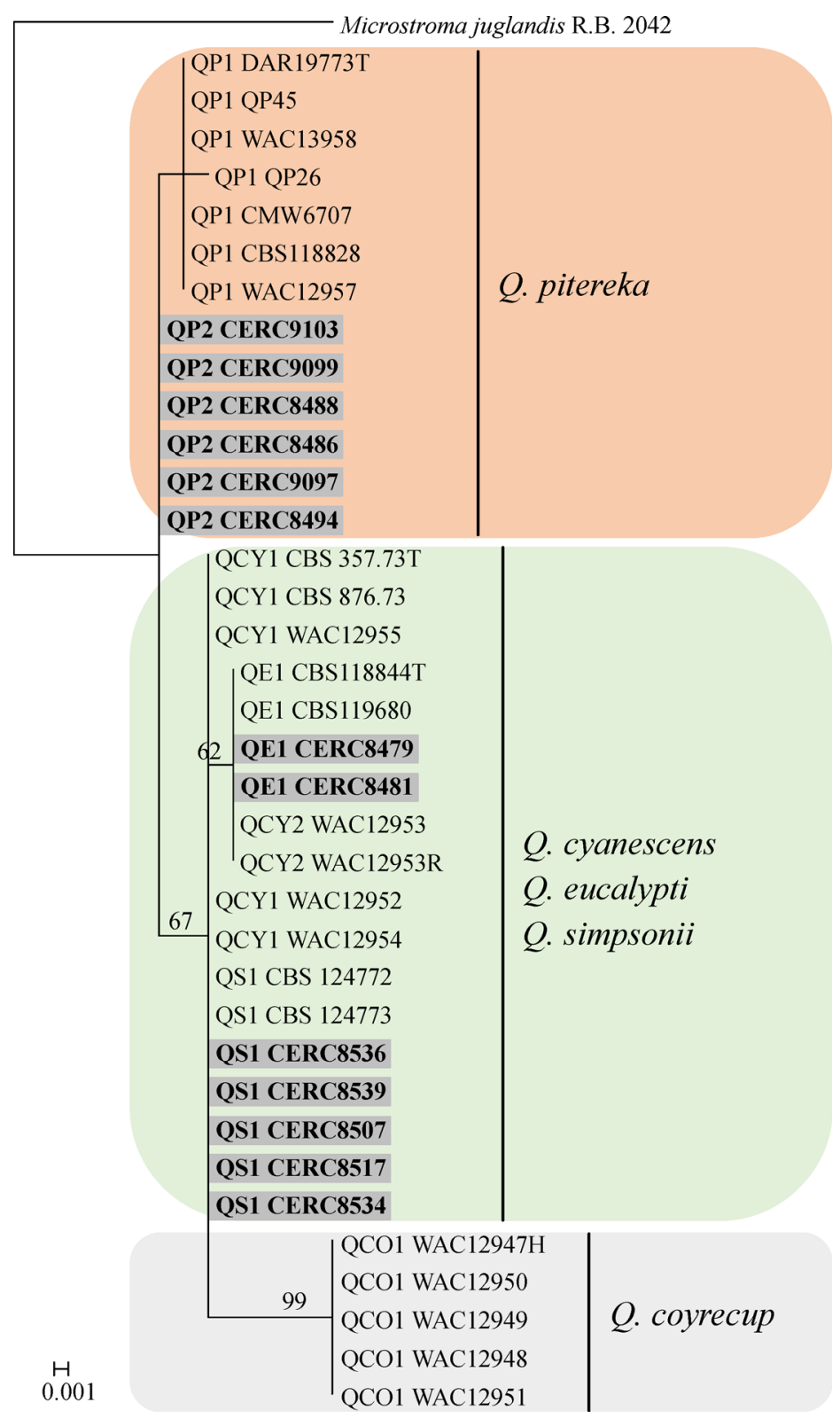

Fig. 3 Phylogenetic tree based on Maximum Likelihood analysis of large subunit sequence data of haplotypes of five Quambalaria species, Q. coyrecup (QCO), Q. cyanescens (QCY), Q. eucalypti (QE), Q. pitereka $(\mathrm{QP})$ and Q. simpsonii (QS), respectively. Bootstrap values $>60 \%$ are presented at branches, bootstrap values $<60 \%$ or absent values are not shown. Haplotypes and isolates from eucalypts in this study are in boldface and highlighted. Isolates representing ex-type are marked with T, isolates repeated are marked with R. The tree is rooted to Microstroma juglandis.

Q. eucalypti (primary conidia ellipsoid, av. $6.2 \mu \mathrm{m} \times 3.8$ $\mu \mathrm{m}$, length/width $=1.6$; secondary conidia obovoid, av. 3.3 $\mu \mathrm{m} \times 2.6 \mu \mathrm{m}$, length $/$ width $=1.3$ ) and $Q$. simpsonii (primary conidia fusiform, av. $7.9 \mu \mathrm{m} \times 3.3 \mu \mathrm{m}$, length/ width $=2.4$; secondary conidia obovoid to ellipsoid, av. 3.7 $\mu \mathrm{m} \times 2.4 \mu \mathrm{m}$, length $/$ width $=1.5$ ), the conidia of $Q$. simpsonii are slight longer and narrower than that of $Q$. eucalypti. The morphology of Q. pitereka, Q. eucalypti and $Q$. simpsonii identified in this study is similar to the results of previous studies ${ }^{[20,23,26]}$.

\section{Discussion}

In this study, three species of Quambalaria, Q. pitereka, $Q$. eucalypti and $Q$. simpsonii, were identified from Eucalyptus and Corymbia plantations in Guangdong and Hainan Provinces in southern China. These Quambalaria spp. were identified and characterized based on phylogenetic analysis of sequence data for LSU and ITS regions, and morphology. This is the first report of $Q$. eucalypti in 

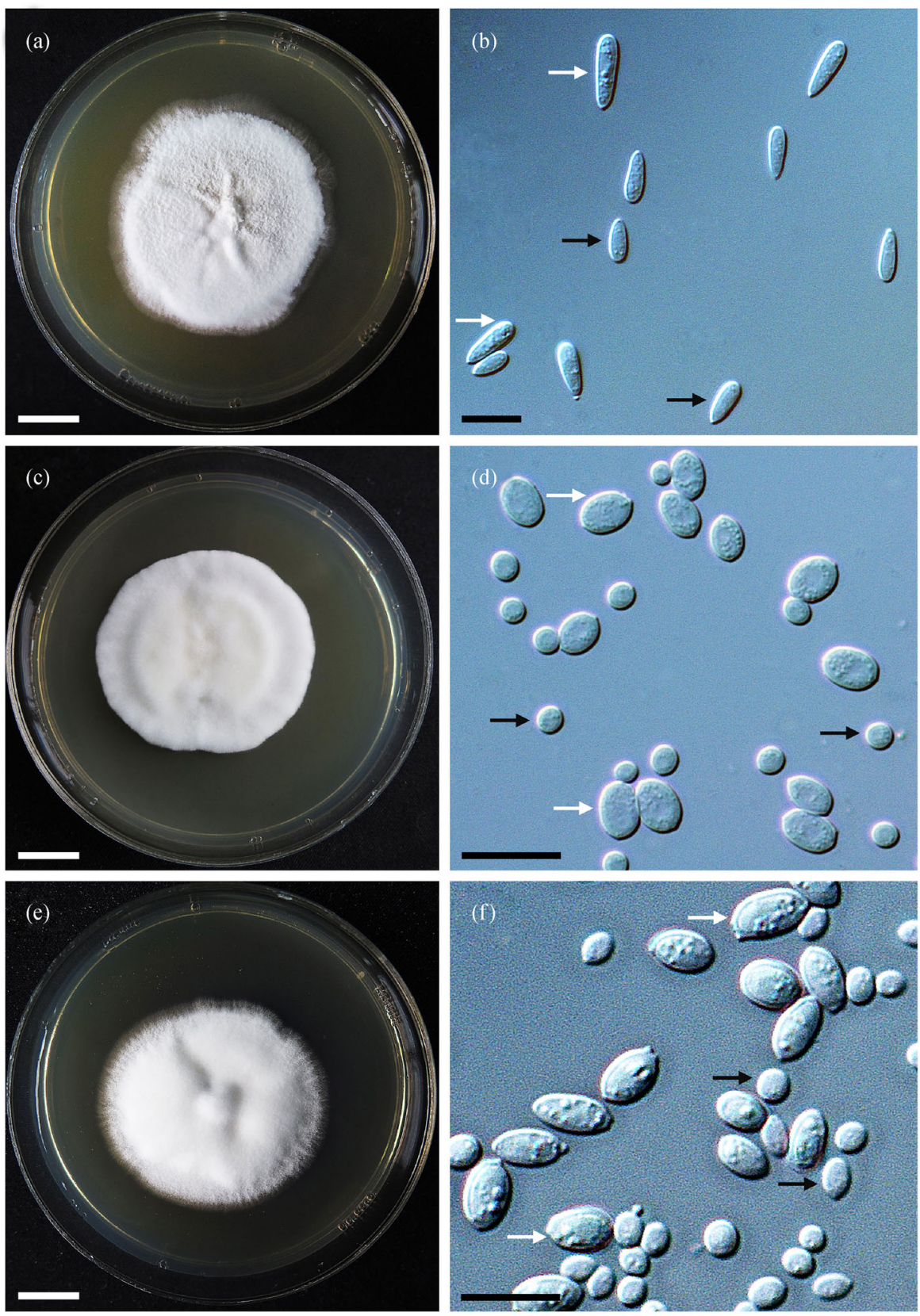

Fig. 4 Cultures grown on malt extract agar at $25^{\circ} \mathrm{C}$ after 2 weeks and the primary and secondary conidia. (a,b) Quambalaria pitereka; (c,d) Q. eucalypti; (e,f) Q. simpsonii.

Asia and the first report of $Q$. simpsonii on eucalypts in China.

Quambalaria pitereka is specific to eucalypts in the genus Corymbia. This fungus is widely distributed in different regions/sites on different species of Corymbia in Australia $^{[24,25]}$. Outside Australia, Q. pitereka has previously been reported only on $C$. citriodora in one plantation in Guangdong Province ${ }^{[17]}$. The results of this study showed that the sequenced isolates of $Q$. pitereka include 12 haplotypes, only one of these was found in China and the remaining haplotypes were known only from Australia. This high level of genetic diversity for isolates from Australia supported the view ${ }^{[24]}$ that $Q$. pitereka was native to that country. In the present study, $Q$. pitereka was isolated from 17 C. citriodora provenances in two experimental plantations. These are relatively distant from the site where $Q$. pitereka was first reported in $2007^{[17]}$ and the ITS haplotype was the same as that found in the study of Zhou et al. ${ }^{[17]}$. These results suggest that $Q$. pitereka could spread actively between different regions and $C$. citriodora provenances in China.

Quambalaria eucalypti is considered to be one of the most important pathogens of eucalypts. Outside Australia, this fungus was first reported on Eucalyptus in nurseries in 


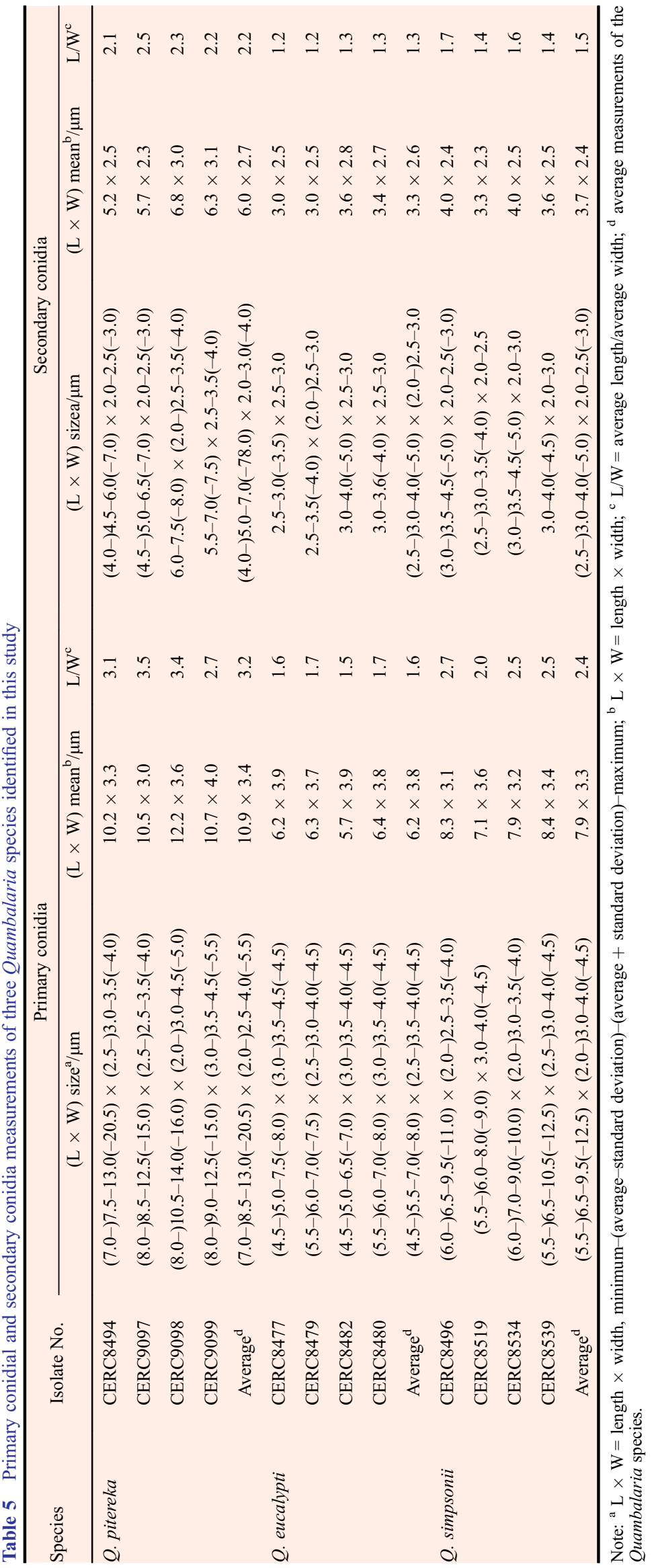


South Africa ${ }^{[20]}$ and it was later found in Brazil ${ }^{[37]}$ and Portugal ${ }^{[27]}$ where it causes leaf spots, shoot infections and lesions on seedling stems. Q. eucalypti has also been recorded in Eucalyptus plantations in Brazil ${ }^{[38]}$, South Africa $^{[30]}$, Australia ${ }^{[24]}$ and Portugal ${ }^{[27]}$ where it can result in severe shoot and leaf blight and stem cankers ${ }^{[24,27,30]}$. Other than on Eucalyptus, Q. eucalypti has been isolated from leaf lesions on native $M$. glaucescens trees in Uruguay $^{[28]}$ and Corymbia species in Australia ${ }^{[24]}$. In this study, $Q$. eucalypti was isolated from a diseased $E$. urophylla $\times E$. grandis clone. It appears to be a pathogen of emerging importance in China.

The ITS haplotype determination showed that all four haplotypes of $Q$. eucalypti determined in this study are found in Australia. Only two of the four haplotypes have been found in other countries including China, Portugal, South Africa and Uruguay. Portugal, South Africa and Uruguay share the same haplotype, the other haplotype apart from Australia was only found in China. Results in this study support the view that $Q$. eucalypti is native to Australia and that this is the source of introductions to new $\operatorname{areas}^{[24]}$

Quambalaria simpsonii was first reported from species of Eucalyptus in Australia and Thailand, but it is unknown whether this is a pathogen ${ }^{[26]}$. In the present study, $Q$. simpsonii was consistently isolated with $T$. zuluensis from cankered $E$. urophylla $\times E$. grandis stems in four sites in Guangdong and Guangxi, China. Whether Q. simpsonii is pathogenic to Eucalyptus trees, and the ecological interaction between $Q$. simpsonii and T. zuluensis remains to be clarified.

\section{Conclusions}

The genus Quambalaria presently includes six species. Most of these are pathogens that cause leaf and shoot blight, and cankers on Eucalyptus and Corymbia. They are considered native to Australia but have been inadvertently introduced into countries of Africa, Asia, Europe and South America. This has most likely occurred via the trade in eucalypt germplasm ${ }^{[39]}$. In the present study, three Quambalaria spp. were identified in China; Q. pitereka on C. citriodora, $Q$. eucalypti on clones $E$. urophylla $\times$ E. grandis and $Q$. simpsonii isolated from stem cankers of $E$. urophylla $\times E$. grandis caused by $T$. zuluensis. These are widespread in areas of China where eucalypts are grown and they are likely to become more important to commercial forestry in the future.

Supplementary materials The online version of this articale at https:// doi. org/10.15302/J-FASE-2017173 contains supplementary material (Table S1).

Acknowledgements The research was financially supported by the Fundamental Research Funds for the Central Non-profit Research Institution of CAF (CAFYBB2014MA018), and the Overseas Outstanding Scholars Lecture Program, the Fundamental Research Funds for the Central Non-profit
Research Institution of CAF (CAFYBB2017ZF005). The authors thank Ms. Jieqiong Li, Ms. Yan Lin and Prof. Jianzhong Luo for their assistance in collecting disease samples in the Guangdong and Hainan Provinces.

Compliance with ethics guidelines Shuaifei Chen, Qianli Liu, Guoqing $\mathrm{Li}$, and Michael J. Wingfield declare they have no conflicts of interest or financial conflicts to disclose.

This article does not contain any studies with human or animal subjects performed by any of the authors.

\section{References}

1. Chen S X, Chen X F. Technical problems and thinking on eucalypt plantation management in China. Eucalypt Science \& Technology, 2013, 30(3): 52-59 (in Chinese)

2. Qi S X. Eucalyptus in China. Bejing: China Forestry House Press, 2002 (in Chinese)

3. Qi S X, Wang H F, Wen Y Q. The Manual of Eucalyptus Plantation. Beijing: China Forestry House Press, 2006 (in Chinese)

4. Xie Y J, Arnold R J, Wu Z H, Chen S F, Du A P, Luo J Z. Advances in eucalypt research in China. Frontiers of Agricultural Science and Engineering , 2017, https:// doi.org/10.15302/J-FASE-2017171

5. Zhou X D, Wingfield M J. Eucalypt diseases and their management in China. Australasian Plant Pathology, 2011, 40(4): 339-345

6. Cortinas M N, Burgess T, Dell B, Xu D, Crous P W, Wingfield B D, Wingfield M J. First record of Colletogloeopsis zuluense comb. nov., causing a stem canker of Eucalyptus in China. Mycological Research, 2006, 110(2): 229-236

7. Chen S F, Barnes I, Chungu D, Roux J, Wingfield M J, Xie Y J, Zhou X D. High population diversity and increasing importance of the Eucalyptus stem canker pathogen, Teratosphaeria zuluensis, in South China. Australasian Plant Pathology, 2011, 40(4): 407-415

8. Chen S F, Pavlic D, Roux J, Slippers B, Xie Y J, Wingfield M J, Zhou X D. Characterization of Botryosphaeriaceae from plantationgrown Eucalyptus species in South China. Plant Pathology, 2011, 60(4): 739-751

9. Chen S F, Gryzenhout M, Roux J, Xie Y J, Wingfield M J, Zhou X D. Identification and pathogenicity of Chrysoporthe cubensis on Eucalyptus and Syzygium spp. in South China. Plant Disease, 2010, 94(9): 1143-1150

10. Chen S, Gryzenhout M, Roux J, Xie Y, Wingfield M J, Zhou X. Novel species of Celoporthe from Eucalyptus and Syzygium trees in China and Indonesia. Mycologia, 2011, 103(6): 1384-1410

11. Chen S F, Van Wyk M, Roux J, Wingfield M J, Xie Y J, Zhou X D. Taxonomy and pathogenicity of Ceratocystis species on Eucalyptus trees in South China, including C. chinaeucensis sp. nov. Fungal Diversity, 2013, 58(1): 267-279

12. Lombard L, Zhou X D, Crous P W, Wingfield B D, Wingfield M J. Calonectria species associated with cutting rot of Eucalyptus. Persoonia, 2010, 24(1): 1-11

13. Lombard L, Chen S F, Mou X, Zhou X D, Crous P W, Wingfield M J. New species, hyper-diversity and potential importance of Calonectria spp. from Eucalyptus in South China. Studies in Mycology, 2015, 80(2): 151-188

14. Chen S F, Lombard L, Roux J, Xie Y J, Wingfield M J, Zhou X D. Novel species of Calonectria associated with Eucalyptus leaf blight 
in Southeast China. Persoonia, 2011, 26(1): 1-12

15. Burgess T I, Andjic V, Hardy G S, Dell B, Xu D. First report of Phaeophleospora destructans in China. Journal of Tropical Forest Science, 2006, 18(2): 144-146

16. Burgess T I, Barber P A, Sufaati S, Xu D, Hardy G S, Dell B. Mycosphaerella spp. on Eucalyptus in Asia: new species, new hosts and new records. Fungal Diversity, 2007, 24(2): 135-157

17. Zhou X D, de Beer Z W, Xie Y J, Pegg G S, Wingfield M J. DNAbased identification of Quambalaria pitereka causing severe leaf blight of Corymbia citriodora in China. Fungal Diversity, 2007, 25 (5): 245-254

18. Walker J, Bertus A L. Shoot blight of Eucalyptus spp. caused by an undescribed species of Ramularia. Proceedings of the Linnean Society of New South Wales, 1971, 96(2): 108-115

19. Bertus A L, Walker J. Ramularia on Eucalyptus and Angophora. Australasian Plant Pathology Society Newsletter, 1974, 3(1): 3

20. Wingfield M J, Crous P W, Swart W J. Sporothrix eucalypti (sp. nov.), a shoot and leaf pathogen of Eucalyptus in South Africa. Mycopathologia, 1993, 123(3): 159-164

21. Simpson J A. Quambalaria, a new genus of eucalypt pathogens. Australasian Mycologist, 2000, 19(2): 57-62

22. de Beer Z W, Begerow D, Bauer R, Pegg G S, Crous P W, Wingfield M J. Phylogeny of the Quambalariaceae fam. nov., including important Eucalyptus pathogens in South Africa and Australia. Studies in Mycology, 2006, 55: 289-298

23. Paap T, Burgess T I, McComb J A, Shearer B L, Hardy G E. Quambalaria species implicated in canker and shoot blight diseases causing decline of Corymbia calophylla and C. ficifolia in the southwest of Western Australia. Mycological Research, 2008, 112 (1): 57-69

24. Pegg G S, O’Dwyer C, Carnegie A J, Burgess T I, Wingfield M J, Drenth A. Quambalaria species associated with plantation and native eucalypts in Australia. Plant Pathology, 2008, 57(4): 702714

25. Pegg G S, Carnegie A J, Wingfield M J, Drenth A. Quambalaria species: increasing threat to eucalypt plantations in Australia. Southern Forests, 2009, 71(2): 111-114

26. Cheewangkoon R, Groenewald J Z, Summerell B A, Hyde K D, ToAnun C, Crous P W. Myrtaceae, a cache of fungal biodiversity. Persoonia, 2009, 23(1): 55-85

27. Braganca H, Diogo E L F, Neves L, Valente C, Araujo C, Bonifacio L, Phillips A J L. Quambalaria eucalypti a pathogen of Eucalyptus globulus newly reported in Portugal and in Europe. Forest Pathology, 2016, 46(1): 67-75

28. Pérez C A, de Beer Z W, Altier N A, Wingfield M J, Blanchette R A. Discovery of the eucalypt pathogen Quambalaria eucalypti infecting a non-Eucalyptus host in Uruguay. Australasian Plant Pathology, 2008, 37(6): 600-604

29. Braun U. A monograph of Cercosporella, Ramularia and allied genera (phytopathogenic hyphomycetes). Volume 2. Munchen: IHW-Verlag, 1998

30. Roux J, Mthalane Z L, de Beer Z W, Eisenberg B, Wingfield M J. Quambalaria leaf and shoot blight on Eucalyptus in South Africa. Australasian Plant Pathology, 2006, 35(4): 427-433

31. van Burik J A H, Schreckhise R W, White T C, Bowden R A, Myerson D. Comparison of six extraction techniques for isolation of DNA from filamentous fungi. Medical Mycology, 1998, 36(5): 299303

32. Chen S F, Wingfield M J, Li G Q, Liu F F. Corticimorbus sinomyrti gen. et sp. nov. (Cryphonectriaceae) pathogenic to native Rhodomyrtus tomentosa (Myrtaceae) in South China. Plant Pathology, 2016, 65(8): 1254-1266

33. Tamura K, Dudley J, Nei M, Kumar S. MEGA4: Molecular Evolutionary Genetics Analysis (MEGA) software version 4.0. Molecular Biology and Evolution, 2007, 24(8): 1596-1599

34. Katoh K, Misawa K, Kuma K, Miyata T. MAFFT: a novel method for rapid multiple sequence alignment based on fast Fourier transform. Nucleic Acids Research, 2002, 30(14): 3059-3066

35. Guindon S, Gascuel O, Rannala B. A simple, fast, and accurate algorithm to estimate large phylogenies by maximum likelihood. Systematic Biology, 2003, 52(5): 696-704

36. Posada D, Crandall K A. MODELTEST: testing the model of DNA substitution. Bioinformatics, 1998, 14(9): 817-818

37. Alfenas A C, Zauza E A V, Rosa O P P, Assis T F. Sporothrix eucalypti a new pathogen of eucalyptus in Brazil. Fitopatologia Brasileira, 2001, 26(2): 221

38. Zauza E A Z, Alfenas A C, Langrell S R H, Tommerup I C. Detection and identification of Quambalaria species in Eucalyptus nurseries and plantations. In: Proceedings of the 8th International Congress of Plant Pathology, Christchurch. Sydney: Horticulture Australia, 2003, 113

39. Burgess T I, Wingfield M J. Pathogens on the move: a 100-year global experiment with planted eucalypts. Bioscience, 2017, 67(1): $14-25$ 